\title{
Automatic Cough Detection in Acoustic Signal using Spectral Features
}

\author{
Renard Xaviero Adhi Pramono, Syed Anas Imtiaz, and Esther Rodriguez-Villegas
}

\begin{abstract}
Cough is a common symptom that manifests in numerous respiratory diseases. In chronic respiratory diseases, such as asthma and COPD, monitoring of cough is an integral part in managing the disease. This paper presents an algorithm for automatic detection of cough events from acoustic signals. The algorithm uses only three spectral features with a logistic regression model to separate sound segments into cough and non-cough events. The spectral features were derived using simple calculation from two frequency bands of the sound spectrum. The frequency bands of interest were chosen based on its characteristics in the spectrum. The algorithm achieved high sensitivity of $90.31 \%$, specificity of $98.14 \%$, and F1-score of $88.70 \%$. Its low-complexity and high detection performance demonstrate its potential for use in remote patient monitoring systems for real-time, automatic cough detection.
\end{abstract}

\section{INTRODUCTION}

Cough is a common symptom that manifests in numerous respiratory diseases [1]. In Chronic Respiratory Diseases (CRDs), such as asthma and Chronic Obstructive Pulmonary Disease (COPD), monitoring of cough is an integral part in managing the disease. In these cases, detecting the occurrence of cough and the onset of their spells, determining the cough frequency, and counting the number of cough events helps to understand the status of the disease progression [2], [3]. Understanding the pattern of cough occurrence over a longer period of time also helps to provide appropriate and timely care to patients.

Manual counting of cough events in recorded sounds, however, is a very laborious and error-prone task and suffers from inter-expert subjectivity in classification. To avoid these problems, there is a growing interest in developing algorithms to automate the process of detecting and counting cough events. A study in [4] used local Hu moment and kNearest Neighbour (KNN) and achieved $88.51 \%$ sensitivity (SE) with specificity (SP) of $99.72 \%$. You et al. [5] employed Support Vector Machine (SVM) with Non-Negative Matrix Factorisation (NMF), and achieved an average performance were SE of $80.1 \%$ and SP of $83.1 \%$. Mel-frequency Cepstral Coefficient (MFCC) has been used by different studies to detect cough sounds. MFCC as a feature for cough detection in [6] coupled with XGBoost algorithm, achieved average Area Under Curve of 0.916 in a Leave-One-Out Cross-Validation (LOOCV) scheme. Matos et al [7] used

This work was supported by the Engineering and Physical Sciences Research Council (EPSRC), UK / grant agreement no. EP/P009794/1. Renard X. A. Pramono gratefully thanks the Indonesian Endowment Fund for Education (LPDP) for its financial support for his study.

R. X. A. Pramono, S. A. Imtiaz, and E. Rodriguez-Villegas are with the Wearable Technologies Lab, Department of Electrical and Electronic Engineering, Imperial College London, SW7 2BT, United Kingdom. Email: \{renard.pramono14, anas.imtiaz, e.rodriguez\}@imperial.ac.uk
MFCC with Hidden Markov Model (HMM) achieving 71\% sensitivity. The study in [8] used Sequential Minimal Optimisation (SMO) with MFCC achieving 75.5\% SE with 99.6\% SP. Liu et al in [9] used Deep Neural Network (DNN) for classification with MFCC feature inputs and achieved performance of $90.1 \%$ SE and $85 \%$ SP. DNN was also used in [10], achieving $86.8 \%$ SE and $92.7 \%$ SP. Drugman et al [11] employed a large set of features including MFCC, spectral flatness, and spectral centroid. These were fed into two different ANNs. After feature selection based on mutual information, 50 features were used for each ANN resulting in classification performance of $89.7 \%$ SE and $89.85 \%$ SP. Another use of ANN was presented in [12], where MFCC, formant frequency, zero crossing rate, non-gaussianity score, and Shannon entropy were used as the input features. The average performance achieved was $92.8 \% \mathrm{SE}$ and $97.5 \%$ SP. Our previous method in [13] used Logistic Regression Model (LRM) with MFCC and spectral features on a 50\%$50 \%$ random data split, where the focus of the study was to diagnose pertussis. Part of the study was detecting coughs achieving a performance of $85.20 \%$ SE and $98.32 \%$ SP.

Most of the works mentioned above either require a large number of features or use complex machine learning methods.In contrast, the objective of this paper is to develop a simpler automatic cough detection method, using only a small number of useful features. This is particularly useful for use in long-term remote monitoring systems for patients with CRDs. This paper proposes a new method of automatic cough detection using only three spectral features, inspired by the observed characteristics of the cough sound spectrum in the frequency domain, and how it differs from other sounds typically recorded when monitoring cough. These characteristics include a wide spread spectrum, low tone prominence, and low harmonicity. To detect such sounds, two frequency bands of interest were extracted from the spectrum, from which the descriptive features were then calculated. Section II describes the database used, preprocessing steps, and the different features extracted from the signals for the algorithm proposed in this paper. Section III introduces the cough detection algorithm and reports its performance. Finally, Section IV discusses these results and compares its performance against those in other studies.

\section{MATERIAL AND METHODS}

\section{A. Database}

For this study, a total of 43 real-world environment recordings, with a length of over 32 minutes, from different patients were obtained from multiple repositories, as described in [13]. Based on the description provided at each source, the 
data collected contained cough sounds from various diseases including pertussis, croup, common cold, bronchitis, bronchiolitis, asthma, and COPD. As the recordings were collected from multiple public repositories, there were variations in the recording instruments, sampling frequencies, and noise level associated with each recording. In these recordings, cough sound locations were manually marked as reference to assess the performance of the proposed cough detection method. As a result, a total of 980 events were labelled as cough sounds whilst more than 1000 separate non-cough sound events were also identified. The non-cough sound events present included speech, laughter, sneeze, throat clearing, wheezing sound, whooping sound, machine noises, and other types of background noise.

\section{B. Preprocessing}

Before being used for analysis, all the recordings were first preprocessed to minimize the variations as a result of different recording conditions. Each recording was first filtered using a 5-th order Butterworth band-pass filter with $70 \mathrm{~Hz}$ and $3500 \mathrm{~Hz}$ as the low and high cut-off frequencies, respectively. All of the recordings were then resampled to $8000 \mathrm{~Hz}$ since cough-related information was observed to be contained below $4000 \mathrm{~Hz}$. Subsequently, the amplitude of the recordings was scaled into a range between -1 and 1 without changing the distribution, so that important statistical parameters of the sounds were preserved. Signals were then segmented into frames of $256 \mathrm{~ms}$ width with $50 \%$ overlap between consecutive segments.

Prior to the detection of cough sounds, it is helpful to remove silent parts of the recordings to ensure that all further processing is performed only on parts of the signals containing a sound event. This also helps in reducing the processing load downstream if the silence removal approach is much simpler than the further processing stages. In this study, silence and low noise frames of the recordings were detected using a simple threshold, based on the standard deviation of the previous samples. Thus, further processing was performed only when the average energy of a frame was above this threshold. Fourier transform was then performed on each detected frame to obtain the frequency spectrum of the signal. Blackman window was used to minimize spectral leakage on the Fourier transform. Each signal frame $x[n]$ was represented by 1025 frequency points between 0 to $4000 \mathrm{~Hz}$ in the spectrum $X(f)$.

\section{Features extraction}

An example of cough and non-cough segment spectrum $X(f)$ is shown in Fig. 1. It can be observed that the spectrum of the cough segment is spread wide, has low tone prominence, and low harmonicity; as mentioned previously. Based on these characteristics, two different frequency bands of interest were extracted. The first frequency band was obtained by taking the frequency contents between the fundamental frequency $F 0$ and its next harmonic $F 1$, called $B-01$. This band was used to represent the low tone prominence and harmonicity of the spectrum. $F 0$ was assumed to be the frequency with the highest spectral content, while $F 1$ was found by finding a peak at frequency near twice $F 0$. The second frequency band edges, called $B-H F$, and used to represent the high frequency component of the spectrum, were 2.5 to $3 \mathrm{kHz}$. An example of these frequency bands for both cough and non-cough segment is shown in Fig. 1. A total of three features were then derived from these frequency bands to detect cough sound as follows.

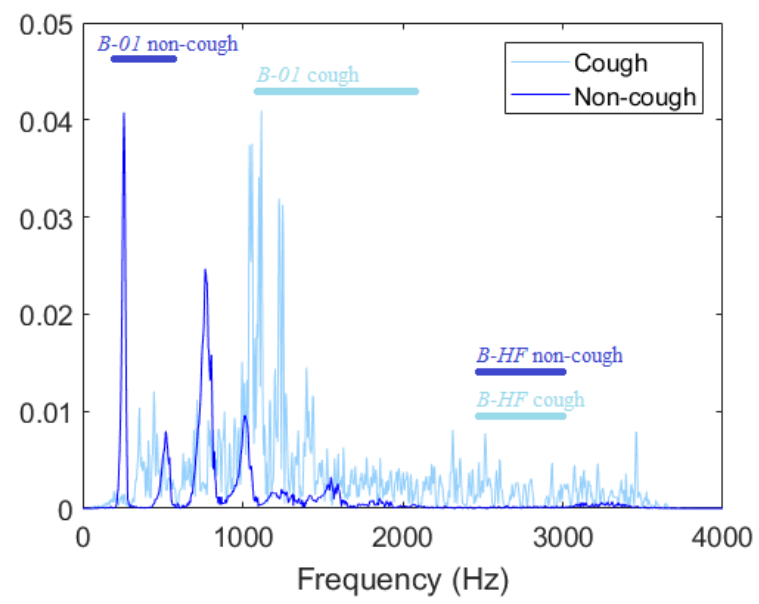

(a)

Fig. 1. Example of spectrum of $256 \mathrm{~ms}$ cough and non-cough segment taken from one recording with their respective frequency bands of interest $B-01$ and $B-H F$.

1) High-frequency content in $B-H F$ ratio: The first feature was calculated using Eq. 1 as the ratio of the median of the high frequency content in $B-H F$ and the spectrum maximum amplitude.

$$
\operatorname{HFMax}_{\text {ratio }}=\frac{\operatorname{median}\left(X(f)_{f \in B-H F}\right)}{\max \left(X(f)_{f \in B-01}\right)}
$$

2) Min-max ratio in $B$-01: The second feature was derived as the ratio between the minimum and maximum value within frequency band $B-01$, as shown in Eq. 2 .

$$
\operatorname{MinMax}_{\text {ratio }}=\frac{\min \left(X(f)_{f \in B-01}\right)}{\max \left(X(f)_{f \in B-01}\right)}
$$

3) Low quantile ratio in $B$-01: The third feature was calculated using Eq. 3. This is the ratio between the median value of the lower quantile and the maximum value in frequency band $B-01$. The lower quantile was defined as in Eq. 4, where the distribution limit is 0.25 .

$$
\begin{gathered}
L Q M_{\text {ratio }}=\frac{\operatorname{median}\left(L Q\left(X(f)_{f \in B-01}\right)\right)}{\max \left(X(f)_{f \in B-01}\right)} \\
L Q(Y)=Y(Y<y), \text { where } \operatorname{Pr}[Y<y] \leq 0.25
\end{gathered}
$$




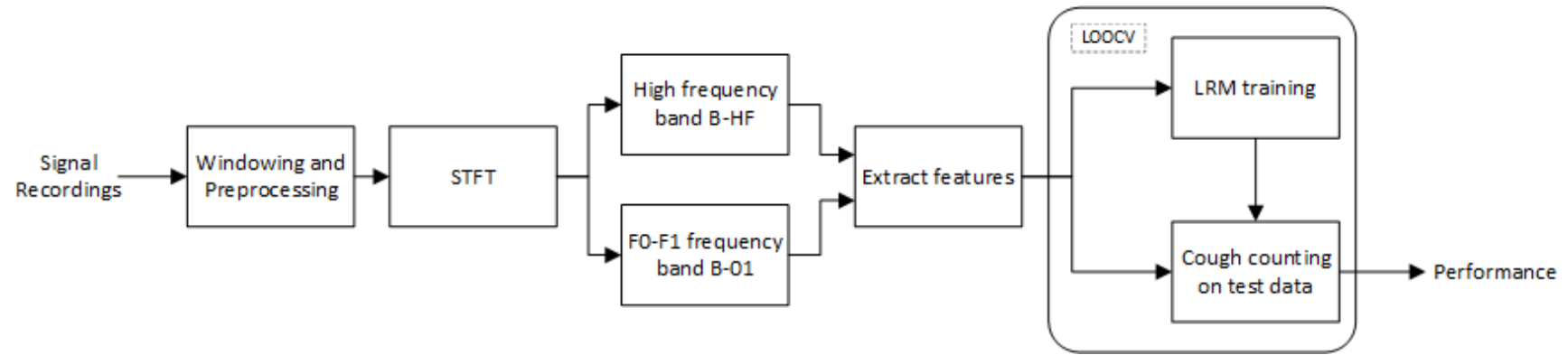

Fig. 2. Overview of the automatic cough detection algorithm.

\section{COUGH SOUND DETECTION ALGORITHM}

\section{A. Overview}

Fig. 2 shows an overview of the proposed cough detection algorithm. The first step was preprocessing, to filter and normalize the sound recordings, as well as to remove silent segments from them. STFT was then used to obtain the spectrum of segments not removed by the pre-processing step. Frequency bands of interest, $B-H F$ and $B-01$, were extracted. The spectral features, HFMax ${ }_{\text {ratio }}$ MinMax ratio $_{\text {, }}$ and $L Q M A X_{\text {ratio }}$, were calculated based on these frequency bands. The features extracted were then scaled into range between -1 and 1 prior to the detection. The segments were then assessed for the presence of cough based on the extracted features using LRM.

To evaluate the detection performance of the algorithm, the recordings were separated into training and test set using LOOCV scheme. The data split was performed at the recording level, such that the training and test set contained different recordings and that the test set consisted of entirely unseen data. Thus, a total of 43 train-test sets were obtained, where the average of the detection performance was reported.

\section{B. Performance metrics}

The performance of the cough detection algorithm was evaluated by counting the number of correctly detected cough events as True Positives (TP), incorrectly missed cough events as False Negatives (FN), correctly rejected noncough frames as True Negatives (TN), and incorrectly labeled non-cough frames as False Positives (FP). From these, the Sensitivity (SE), Specificity (SP), Positive Predictive Value (PPV), Negative Predictive Value (NPV), F1-score (F1), and Matthews Correlation Coefficient (MCC) were calculated, as in Eq. 5.

$$
\begin{gathered}
S E=\frac{T P}{T P+F N}, S P=\frac{T N}{T N+F P}, \\
P P V=\frac{T P}{T P+F P}, N P V=\frac{T N}{T N+F N}, \\
F 1=\frac{2(S E)(P P V)}{S E+P P V}, \\
M C C=\frac{(T P)(T N)-(F P)(F N)}{\sqrt{(T P+F P)(T P+F N)(T N+F P)(T N+F N)}}
\end{gathered}
$$

TABLE I

Average Detection Performance

\begin{tabular}{lclc}
\hline Metric & Average (\%) & Metric & Average (\%) \\
\hline SE & 90.31 & PPV & 88.47 \\
SP & 98.14 & NPV & 98.54 \\
F1 & 88.70 & MCC & 87.46 \\
\hline
\end{tabular}

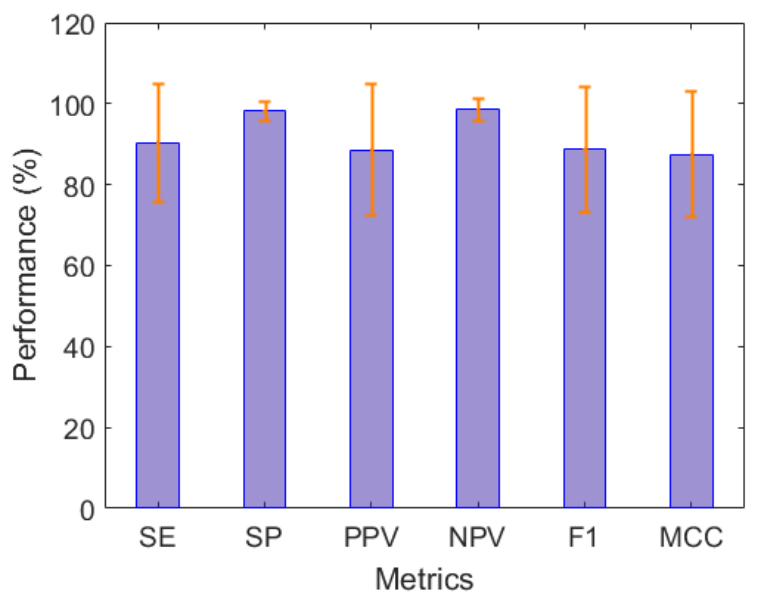

Fig. 3. Performance of the cough detection algorithm.

\section{Results}

In each repetition, the training set consisted of 42 recordings which were used to train the LRM while the one recording left was used as the test set to evaluate the model. The evaluation performance in terms of TP, TN, FP, and FN of each repetition was obtained, from which SE, SP, PPV, NPV, F1, and MCC were calculated. Table I shows the cough detection algorithm LOOCV average performance, using the proposed features. The standard deviation of each metric is shown in Fig. 3. It can be observed, from Table I, that the average SE achieved was $90.31 \%$ while the average PPV, F1, and MCC was $88.47 \%, 88.70 \%$, and $87.46 \%$ respectively. The high sensitivity shows that most cough events were detected, while the low false positive detection resulted in a high PPV, F1, and MCC. The specificity and NPV values are also high, showing the ability of the algorithm to reject most non-cough segments correctly. 
TABLE II

Detection PERFormance COMPARISON

\begin{tabular}{|c|c|c|c|c|c|c|c|c|c|}
\hline Ref. & SE (\%) & SP (\%) & PPV (\%) & NPV (\%) & F1 (\%) & $\operatorname{MCC}(\%)$ & Features Type & \# Features & Method \\
\hline [4] & 88.51 & 99.72 & 87.51 & 99.78 & 88.02 & 86.85 & Cepstral & 13 & $\mathrm{kNN}$ \\
\hline [5] & 80.10 & 83.10 & 83.50 & - & 81.76 & - & Spectral & 60 & SVM \\
\hline [6] & AUC & 91.60 & - & - & - & - & Cepstral & 12 & XGBoost \\
\hline [7] & 71.00 & FAR $13 / \mathrm{h}$ & - & - & - & - & Cepstral & 39 & HMM \\
\hline [8] & 75.50 & 99.60 & - & - & - & - & Cepstral & 39 & SMO \\
\hline$[9]$ & 90.10 & 85.50 & _ & ـ & 40.00 & - & Cepstral & 39 & DNN \\
\hline$[10]$ & 86.80 & 92.70 & - & - & - & - & Spectral & 64 & $\mathrm{CNN}$ \\
\hline [11] & 89.70 & 89.85 & - & - & - & - & Cepstral, Spectral, Time & 50 & ANN \\
\hline$[12]$ & 92.80 & 97.50 & - & - & - & - & Cepstral, Spectral, Time & 22 & TDNN \\
\hline [13] & 85.20 & 98.32 & 84.62 & 98.37 & 84.91 & 83.16 & Cepstral, Spectral, Time & 26 & LRM \\
\hline This work & 90.31 & 98.14 & 88.47 & 98.54 & 88.70 & 87.46 & Spectral & 3 & LRM \\
\hline
\end{tabular}

\section{DISCUSSION}

In this paper, a new method for automatic detection of cough events is presented. The method uses only three spectral features with a logistic regression model for classification of acoustic sound segments as cough or non-cough events. The performance of the algorithm was evaluated on a database that consisted of 980 cough sounds and more than 1000 non-cough events, from 43 different recordings. Using LOOCV as validation scheme, consistent results were obtained with high average sensitivity of $90.31 \%$ and PPV of $88.47 \%$. Performance of the algorithm is also reported as F1-score to show the low rate of false positives and MCC where a more balanced ratio of the positive and negative classes can be obtained. The algorithm proposed achieved F1-score and MCC of $88.70 \%$ and $87.46 \%$, respectively. The small range of performance variation in each iteration demonstrates the capability of the proposed algorithm to work well with unseen data. It also shows that the small number of features used in this work compared to previous studies provide enough information that can be used for a reliable detection of cough sounds.

Table II shows the comparison of the cough detection performance between the proposed algorithm and those in other studies. It can be seen that, of the ten other listed studies, only [12] have reported SE higher than what is achieved in this paper. However, the proposed algorithm still achieves higher SP while using less features when compared to this study. Additionally, the methods presented in the other studies in Table II are more complex than the algorithm in this paper. For example [4], uses kNN classification with local $\mathrm{Hu}$ moment as the main feature which, as reported by the authors, is very time-consuming to compute. The algorithm in [9] uses a deep neural network for classification with 13 MFCC features, while [12] also uses a neural network with MFCC, with additional time and frequency features. In contrast, the algorithm proposed in this paper uses a simple classifier with only three features and achieves a comparable detection performance. It should be noted though that the data used to report performances of the different algorithms is not the same. The differences in signal acquisition methods, recording conditions, and types of noncough signals present in different data would have an impact on the achieved performance. However, since the data used by most studies is not publicly available, it is not possible to make a direct comparison between all the different methods. Nevertheless, the results reported in this study demonstrate the potential for the proposed low-complexity algorithm to be used in remote patient monitoring systems for real-time, automatic cough detection.

\section{REFERENCES}

[1] A. B. Goldsobel and B. E. Chipps, "Cough in the pediatric population," The Journal of pediatrics, vol. 156, no. 3, pp. 352-358, 2010.

[2] British Thoracic Society and Scottish Intercollegiate Guidelines Network, "British guideline for the management of asthma; a national clinical guideline (sign 153)," September 2016, last accessed 07 February 2018. [Online]. Available: https://www.britthoracic.org.uk/guidelines-and-quality-standards/asthma-guideline/

[3] — - "Chronic obstructive pulmonary disease in over 16s: diagnosis and management," June 2010, last accessed 07 February 2018. [Online]. Available: https://www.nice.org.uk/guidance/CG101

[4] J. Monge-Alvarez, C. Hoyos-Barcelo, P. Lesso, and P. Casaseca-dela Higuera, "Robust detection of audio-cough events using local hu moments," IEEE Journal of Biomedical and Health Informatics, 2018.

[5] M. You, H. Wang, Z. Liu, C. Chen, J. Liu, X.-H. Xu, and Z.-M. Qiu, "Novel feature extraction method for cough detection using nmf," IET Signal Processing, vol. 11, no. 5, pp. 515-520, 2017.

[6] L. Di Perna, G. Spina, S. Thackray-Nocera, M. G. Crooks, A. H. Morice, P. Soda, and A. C. den Brinker, "An automated and unobtrusive system for cough detection," in Life Sciences Conference (LSC), 2017 IEEE. IEEE, 2017, pp. 190-193.

[7] S. Matos, S. S. Birring, I. D. Pavord, and H. Evans, "Detection of cough signals in continuous audio recordings using hidden markov models," IEEE Transactions on Biomedical Engineering, vol. 53, no. 6, pp. 1078-83, 062006.

[8] S. Larson, G. Comina, R. H. Gilman, B. H. Tracey, M. Bravard, and J. W. López, "Validation of an automated cough detection algorithm for tracking recovery of pulmonary tuberculosis patients," PloS one, vol. 7, no. 10, p. e46229, 2012.

[9] J.-M. Liu, M. You, Z. Wang, G.-Z. Li, X. Xu, and Z. Qiu, "Cough detection using deep neural networks," in 2014 IEEE International Conference on Bioinformatics and Biomedicine (BIBM), Dept. of Control Sci. Eng., Tongji Univ., Shanghai, China. Piscataway, NJ, USA: IEEE, 2-5 Nov. 2014 2014, pp. 560-3.

[10] J. Amoh and K. Odame, "Deep neural networks for identifying cough sounds," IEEE transactions on biomedical circuits and systems, vol. 10, no. 5, pp. 1003-1011, 2016.

[11] T. Drugman, J. Urbain, N. Bauwens, R. Chessini, A.-S. Aubriot, P. Lebecque, and T. Dutoit, "Audio and contact microphones for cough detection," in Thirteenth Annual Conference of the International Speech Communication Association, 2012.

[12] Y. A. Amrulloh, U. R. Abeyratne, V. Swarnkar, R. Triasih, and A. Setyati, "Automatic cough segmentation from non-contact sound recordings in pediatric wards," Biomedical Signal Processing and Control, vol. 21, pp. 126-136, 2015.

[13] R. X. A. Pramono, S. A. Imtiaz, and E. Rodriguez-Villegas, "A cough-based algorithm for automatic diagnosis of pertussis," PloS one, vol. 11, no. 9, p. e0162128, 2016. 